\title{
A NOTE ON PUSHDOWN STORE AUTOMATA AND REGULAR SYSTEMS ${ }^{1}$
}

\author{
SHEILA A. GREIBACH
}

Recent work on pushdown store automata has focused attention on various sets of pushdown store tapes [8]. Certain sets of tapes associated with pushdown store automata can be proved regular. As a consequence we obtain a new proof of a theorem due to Büchi: ${ }^{2}$ that regular canonical systems (i.e., productions of the form $\alpha Q \rightarrow \beta Q$ ) produce regular sets $[2] .^{3}$

In this paper we shall use a theorem of Bar-Hillel, Perles and Shamir [1] to show that the set of tapes left on the pushdown store by a regular set is regular, ${ }^{4}$ and derive Büchi's theorem from that result.

First we shall need some definitions. We assume familiarity with the definition of production systems. ${ }^{5}$

Definition. A finite state grammar is a quadruple $G=(I, T, X, P)$, where $I$ and $T$ are finite sets, $I \cap T=\varnothing, X \in I$ and $P$ is a finite set of semi-Thue productions of the forms

$$
\mathbf{Q}_{1} Z Q_{2} \rightarrow Q_{1} a Y Q_{2}, \quad Q_{1} Z Q_{2} \rightarrow Q_{1} a Q_{2}, \quad Z, Y \in I, a \in T \cup\{\lambda\} .
$$

A set $L$ is regular iff $L=\left\{w \in T^{*} \mid X \Longrightarrow * w\right\}$ for some finite state grammar $G .^{6}$

We must now define pushdown store automata and their actions.

Received by the editors January 17, 1966.

1 The research reported in this paper was sponsored in part by the Air Force Cambridge Research Laboratories, Office of Aerospace Research, under Contract AF 19(682)-5166, CRL-Algorithmic Languages Program.

${ }^{2}$ Büchi's proof is more elementary; the proof in this paper is shorter and less complicated because it follows from other results.

${ }^{3}$ This theorem is not to be confused with Chomsky's observation that finite state grammars generate regular sets [3], or the theorem of Evey [6] and Matthews [13] that left generations of semi-Thue systems produce context-free sets; the systems involved are different in form.

4 This result is part of the folklore on the subject, but, as far as this author is aware, has never appeared in print. Analogous theorems are proven in [8] and [9] by different methods; the present approach could have been used in [8].

5 See [5], [14], [15] and [17] for further discussion of productions and combinatorial systems.

Notation. If $Q_{1} \alpha Q_{2} \rightarrow Q_{1} \beta Q_{2}$ is a semi-Thue production, we write $w_{1} \alpha w_{2} \Rightarrow w_{1} \beta w_{2}$ for any strings $w_{1}, w_{2} . \Rightarrow *$ denotes the transitive closure of $\Rightarrow$.

' For any set $R$, the closure of $R$, denoted by $R^{*}$, is the free semigroup (with identity $\lambda$ ) generated by $R$.

Justification for this definition appears in [1] and [3]; different but equivalent characterizations of regular sets appear in [2], [12] and [16]. 
Definition. A pushdown store automaton (pda) is a septuple $M$ $=\left(K, \Sigma, \Gamma, \delta, q_{0}, \$, F\right)$, where (1) $K, \Sigma, \Gamma$ are finite sets, $\$ \in \Gamma, F \subseteq K$, (2) $\delta$ is a function from $K x(\Sigma \cup\{\lambda\}) x \Gamma$ in to the finite subsets of $K x \Gamma^{*}$.

$$
\begin{aligned}
\left(q, a y, A w^{\prime}\right) \vdash_{M}\left(q^{\prime}, y, w w^{\prime}\right) \text { if }\left(q^{\prime}, w\right) & \in \delta(q, a, A), \\
w^{\prime} & \in \Gamma^{*}, a \in \Sigma \cup\{\lambda\},
\end{aligned}
$$

$A \in \Gamma$ and $y \in \Sigma^{*}$. $\vdash_{M}^{*}$ is the transitive closure of $\vdash_{M}{ }^{7}$ Null $(M)$ $=\left\{w \mid \exists q \in F,\left(q_{0}, w, \$\right) \vdash_{M}^{*}(q, \lambda, \lambda)\right\}$.

Intuitively, Null $(M)$ is the set of all input tapes that empty the pushdown store and cause the pda to enter a final state at the end.

Definition. $L$ is context-free iff $L=\operatorname{Null}(M)$ for some pda $M{ }^{8}$

In order to state the necessary results clearly, we give the following definitions.

DEFinition. $L$ is a $u c v$-language iff for some finite vocabulary $T$ and some $c \notin T, L \subseteq T^{*} c T^{*}$. If $L$ is a $u c v$-language, let

$$
\begin{array}{ll}
f_{L}(u)=\{v \mid u c v \in L\}, & g_{L}(v)=\{u \mid u c v \in L\}, \\
U(L)=\left\{u \mid f_{L}(u) \neq \varnothing\right\}, & V(L)=\left\{v \mid g_{L}(v) \neq \varnothing\right\} .
\end{array}
$$

We can now state the relevant theorem of Bar-Hillel, Perles and Shamir [1] as:

THEOREM 1. Let $L$ be a context-free ucv-language. If for every $u$, $f_{L}(u)$ is finite, then $V(L)$ is regular. If for every $v, g_{L}(v)$ is finite, then $U(L)$ is regular.

We now focus attention on the tapes left on the pushdown store when reading any member of a given regular set and ending in a given state.

Theorem 2. Let $M:\left(K, \Sigma, \Gamma, \delta, q_{0}, \$, F\right)$ be a pda. Let $q \in K$, and let $R \subseteq \Sigma^{*}$ be regular. Then

$$
V_{q}=\left\{u \mid \exists w \in R,\left(q_{0}, w, \$\right) \vdash_{M}^{*}(q, \lambda, u)\right\}
$$

is regular.

Proof. Let $c$ be a new symbol. First we shall see that

7 The notation used here is the reverse of that employed by the author elsewhere [8]; here we read pushdown store tapes from left to right for convenience; if we considered productions $Q \alpha \rightarrow Q \beta$, the other notation would be preferable.

${ }^{8}$ Context-free languages are usually defined by special semi-Thue systems; see [1] or [3]. The equivalence of the present definition to the standard one can be easily obtained as a corollary to results in [4] and [6]; a particularly clean proof appears in [7]. 


$$
L_{1}=\left\{w c u \mid\left(q_{0}, w, \$\right) \vdash_{M}^{*}(q, \lambda, u)\right\}
$$

is context-free. We modify the pda $M$ to produce a pda $M_{1}$, which imitates $M$ unless and until it sees $c$ in state $q$. Then it empties the pushdown store, checking against the input tape. Clearly $M_{1}$ can be constructed, Null $\left(M_{1}\right)=L_{1}$ and $V_{q}=V\left(L_{1} \cap R c \Gamma^{*}\right)$. If $f_{L_{1}}(w)$ is finite for all $w \in R$, we are done. But the $\lambda$-rules may allow $M$ infinitely many actions on one input tape and hence one input might leave infinitely many distinct tapes on the pushdown store. So, instead of $\lambda$-rules, we use a dummy symbol (or "clock pulse") $d \notin \Sigma \cup \Gamma \cup\{c\}$. $M_{1}$ is modified to produce a pda $M_{2}$ which behaves like $M_{1}$, except that where, for $s$ in $K$ and $A$ in $\Gamma$,

$$
\delta(s, \lambda, A)=\delta_{1}(s, \lambda, A) \neq \varnothing
$$

we have in $M_{2}$ :

$$
\delta_{2}(s, \lambda, A)=\varnothing \quad \text { and } \quad \delta_{2}(s, d, A)=\delta(s, \lambda, A) .
$$

Let $L_{2}=\operatorname{Null}\left(M_{2}\right)$. Let $\psi(a)=a$ for $a \neq d$ and $\psi(d)=\lambda$. Let $L_{3}=L_{2}$ $\cap\left(\psi^{-1}\left(R c \Gamma^{*}\right)\right)$. Clearly, $V_{q}=V(L)_{z}$, and for each $w, f_{L_{\mathbf{z}}}(w)$ is finite. $L_{2}$ is context-free. The inverse of a homomorphism preserves regularity [10], and the intersection of a context-free language and a regular language is context-free [1]. Hence $L_{3}$ is context-free and, by Theorem $1, V_{q}=V\left(L_{3}\right)$ is regular.

Now we must define regular canonical systems to derive the desired results.

Definition. A regular canonical system is a quintuple $R$ $=(I, T, U, V, P)$, where

(1) $I$ and $T$ are finite sets and $I \cap T=\varnothing$,

(2) $U, V \subseteq(I \cup T)^{*}$, and

(3) $P$ is a finite set of regular productions of the form

$$
\alpha Q \rightarrow \beta Q \quad \alpha, \beta \in(I \cup T)^{*} .
$$

Notation. When treating a regular canonical system $R$ we shall write $R$-deductions as $u \Rightarrow_{p} v$ or $u \Rightarrow_{p}^{*} v .^{9}$

Definition. Let $R=(I, T, U, V, P)$ be a regular canonical system. The set $\tau(U, P, V)$ of words produced by $R$ is

$$
\tau(U, P, V)=\left\{x \in T^{*} \mid \exists u \in U, v \in V, u \Rightarrow_{p}^{*} v x\right\} .
$$

The set $\beta(U, P, V)$ of words accepted by $R$ is

\footnotetext{
9 If $\alpha Q \rightarrow \beta Q$ is in $P$, then $\alpha u \Rightarrow_{p} \beta u$ for any $u \in(I \cup T)^{*}$. If $w_{i} \Rightarrow_{p} w_{i+1}$ for $1 \leqq i<n$, then $w_{1} \Rightarrow w_{n}$.
} 


$$
\beta(U, P, V)=\left\{x \in T^{*} \mid \exists u \in U, v \in V, u x \Rightarrow_{p}^{*} v\right\} .
$$

$\tau(U, P,\{\lambda\})$ is the set of theorems of $R$.

Büchi showed that if $U$ is finite, then $\tau(U, P,\{\lambda\})$ is regular and, moreover, all regular sets can be obtained in this fashion [2]. We shall see that, if $U, V$ are any regular sets over $I \cup T$, then $\tau(U, P, V)$ and $\beta(U, P, V)$ are regular.

Notation. If $a$ is an individual symbol, $\breve{a}=a ; \check{\lambda}=\lambda$. If $x=A_{1} \cdots A_{m}$ is a string, then $\check{x}=\breve{A}_{m} \cdots \breve{A}_{1}$. If $S$ is a set, $\check{S}=\{x \mid x \in S\} . S$ is regular iff $\breve{S}$ is regular [16].

Theorem 3. Let $R=(I, T, U, V, P)$ be a regular canonical system. Let $U$ and $V$ be regular sets (finite or infinite). Then $\tau(U, P, V)$ is regular.

Proof. We now construct a special pda $M$ that accepts members of $(I \cup T)^{*}$ as input, places them (reversed) on the pushdown store, and proceeds to imitate the deductions of $R$.

Let $n=\operatorname{Max}\{l(\alpha) \mid \exists \beta, \alpha Q \rightarrow \beta Q \in P\} .{ }^{10}$ Let $M=\left(K, \Sigma, \Gamma, \delta, q_{0}, \$, F\right)$. We define:

$K=\left\{q(w) \mid w \in(I \cup T)^{*}, 0 \leqq l(w) \leqq n\right\} \cup\left\{q_{0}, q_{f}\right\}$, each $q(w)$ a new symbol,

$\Gamma=I \cup T \cup\{\$\}, \$$ a symbol not in $I \cup T$,

$\Sigma=I \cup T$,

$F=\left\{q_{f}\right\}$.

$\delta$ is defined in the following parts:

(I) For all $A \in I \cup T, B \in I \cup T \cup\{\$\}$, we set

$$
\begin{aligned}
\delta\left(q_{0}, A, B\right) & =\left\{\left(q_{0}, A B\right),(q(\lambda), A B)\right\} . \\
\delta\left(q_{0}, \lambda, \$\right) & =\{(q(\lambda), \$)\} .
\end{aligned}
$$

(II) For each $A \in I \cup T$ and $w \in(I \cup T)^{*}$, with $0 \leqq l(w)<n$,

$$
(q(w A), \lambda) \in \delta(q(w), \lambda, A) .
$$

(III) For each $\alpha Q \rightarrow \beta Q$ in $P$ and each $A \in I \cup T \cup\{\$\}$,

$$
(q(\lambda), \beta A) \in \delta(q(\alpha), \lambda, A) .
$$

(IV) For each $A \in I \cup T \cup\{\$\}$,

$$
\left(q_{f}, A\right) \in \delta(q(\lambda), \lambda, A) .
$$

The parts of the pda work as follows:

${ }^{10} l(\alpha)$ is the length of the string $\alpha, l(\lambda)=0$. 
(I) $\left(q_{0}, \check{u}, \$\right) \vdash_{M}^{*}(q(\lambda), \lambda, u \$)$ for $u \in(I \cup T)^{*}$.

(II) $(q(\lambda), \lambda, w y) \vdash_{M}^{*}(q(w), \lambda, y)$ iff $0 \leqq l(w) \leqq n$.

(III) $(q(\alpha), \lambda, y) \vdash_{M}(q(\lambda), \lambda, \beta y)$ iff $\alpha Q \rightarrow \beta Q$ is in $P$.

(IV) $(q(\lambda), \lambda, w \$) \vdash M\left(q_{f}, \lambda, w \$\right)$.

Putting this together we get

$$
\left(q_{0}, \check{u}, \$\right) \vdash_{M}^{*}(q(\lambda), \lambda, u \$) \vdash_{M}^{*}(q(\lambda), \lambda, v \$) \vdash_{M}\left(q_{f}, \lambda, v \$\right) \quad \text { iff } u \Rightarrow_{p}^{*} v \text {. }
$$

Since $U$ is regular, so is $\check{U}$. By Theorem 2 , the set

$$
V_{q_{f}}=\left\{v \mid \exists u \in, \check{U}\left(q_{0}, u, \$\right) \vdash_{M}^{*}\left(q_{f}, \lambda, v\right)\right.
$$

is regular. This yields

$$
V_{q_{f}}=\left\{x \mid \exists u \in U, u \Rightarrow_{p}^{*} x\right\} .
$$

Since the quotient of regular sets is regular [11], then

$$
\tau(U, P, V)=\left\{x \mid \exists v \in V, v x \$ \in V q_{f}\right\} \cap T^{*}
$$

is regular.

Corollary. Let $R=(I, T, U, V, P)$ be a regular canonical system. Let $U$ and $V$ be regular sets. Then $\beta(U, P, V)$ is regular.

Proof. Let $\hat{P}$ be the set of regular productions defined as follows:

$$
\hat{P}=\{\beta Q \rightarrow \alpha Q \mid \alpha Q \rightarrow \beta Q \in P\}
$$

and let $\hat{R}$ be the regular canonical system, $R=(I, T, U, V, \hat{P})$. Then clearly $\beta(U, P, V)=\tau(V, \hat{P}, U)$, so that $\beta(U, P, V)$ is regular by Theorem 3 .

REMARKs. Obviously we have the same results for reverse regular canonical systems whose productions have the form $Q \alpha \rightarrow Q \beta$.

Instead of appealing to the quotient theorem in the proof of Theorem 3, we could have had $M$ delete some member of $V$ from the pushdown store before going to $q_{f}$; from this we could have easily obtained another proof of the quotient theorem for regular sets.

\section{REFERENCES}

1. Y. Bar-Hillel, M. Perles and E. Shamir, On formal properties of simple phrase structure grammars, $Z$. Phonetik Sprachwiss. Kommunikat. 14 (1961), 143-172.

2. J. R. Büchi, Regular canonical sysetms, Arch. Math. Logik Grundlagenforsch 6 (1964), 91-111.

3. N. Chomsky, On certain formal properties of grammars, Information and Control 2 (1959), 137-167. 
4. - Context-free grammars and pushdown storage, Quarterly Progress Report No. 65, Research Laboratory of Electronics, Massachusetts Institute of Technology, Cambridge, Mass., 1962.

5. M. Davis, Computability and unsolvability. McGraw-Hill, New York, 1958.

6. J. Evey, The theory and applications of pushdown store machines, Ph.D. Thesis, Harvard University, Cambridge, Mass., 1963.

7. S. Ginsburg, The mathematical theory of context-free languages, McGraw-Hill, New York, 1966.

8. S. Ginsburg and S. Greibach, Deterministic context-free languages, Information and Control 9 (1966), 620-648.

9. S. Ginsburg, S. Greibach and M. Harrison, Stack automata and compiling, J. Assoc. Comput. Mach. 14 (1967), 172-201.

10. S. Ginsburg and G. F. Rose, Operations which preserve definability in languages, J. Assoc. Comput. Mach. 10 (1963), 175-195.

11. S. Ginsburg and E. Spanier, Quotients of context-free languages, J. Assoc. Comput. Mach. 10 (1963), 487-492.

12. S. C. Kleene, Representalion of events in nerve nets and finite automata, Automata Studies, (C. E. Shannon and J. McCarthy, Eds.), pp. 3-40, Princeton Univ. Press, Princeton, N. J., 1956.

13. G. H. Matthews, $A$ note on asymmetry in phrase structure grammars, Information and Control 7 (1964), 360-365.

14. E. L. Post, Formal Reductions of the general combinatorial decision problem, Amer. J. Math. 65 (1943), 197-215.

15. - Recursive unsolvability of a problem of Thue, J. Symbolic Logic 12 (1947), 1-11.

16. M. Rabin and D. Scott, Finite automata and their decision problems, IBM J. Res. Develop. 3 (1959), 114-125.

17. A. Thue, Probleme über Veränderungen von Zeichenreihen nach gegebenen Regeln, Skr. Vid.-Selskapet Kristiania. I, (1914), no. 10, 1-34.

System Development Corporation, Santa Monica, California and HARVARD UNIVERSITY 